\title{
Virtual care has potential to fragment primary care and disturb continuity of care, warn doctors
}

Cite as: CMAJ 2019 September 16;191:E1038-9. doi: 10.1503/cmaj.1095796

Posted on cmajnews.com on August 29, 2019.

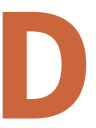

octors in Ontario are concerned about the growth of virtual care walk-in clinics and are calling on the province's government to support family doctors in offering virtual care for rostered patients.

The company Good Doctors has 66 clinics listed on its website, most located in rural communities. The clinics are often located in pharmacies, where a registered nurse or trained assistant guides the patient and conducts tests, directed by a doctor via video.

"The goal of Good Doctors is to be able to get in communities where there is maybe 100 people in town, perhaps no road access," said Leo Liao, the company's director, adding that about half of patients who use the service don't have family doctors. Good Doctors also has clinics in larger communities that are "very, very short on doctors."

Dr. Veronica Legnini, a family physician in Kingston, is concerned, however, that virtual walk-in clinics are opening in areas that have enough doctors. One of her patients was diagnosed with a chest infection at such a clinic, which he went to because it was closer, according to Legnini. When the patient came to see her a week later, she diagnosed him with severe angina. "He could have died," she said. She worries the growth of virtual walk-in clinics is leading to incorrect diagnoses, unnecessary antibiotic prescriptions and duplication of care.

Dr. Sarah Newbery, a family physician in Marathon, Ontario, says another problem with virtual care walk-in clinic is the extra work they create for rural doctors. In

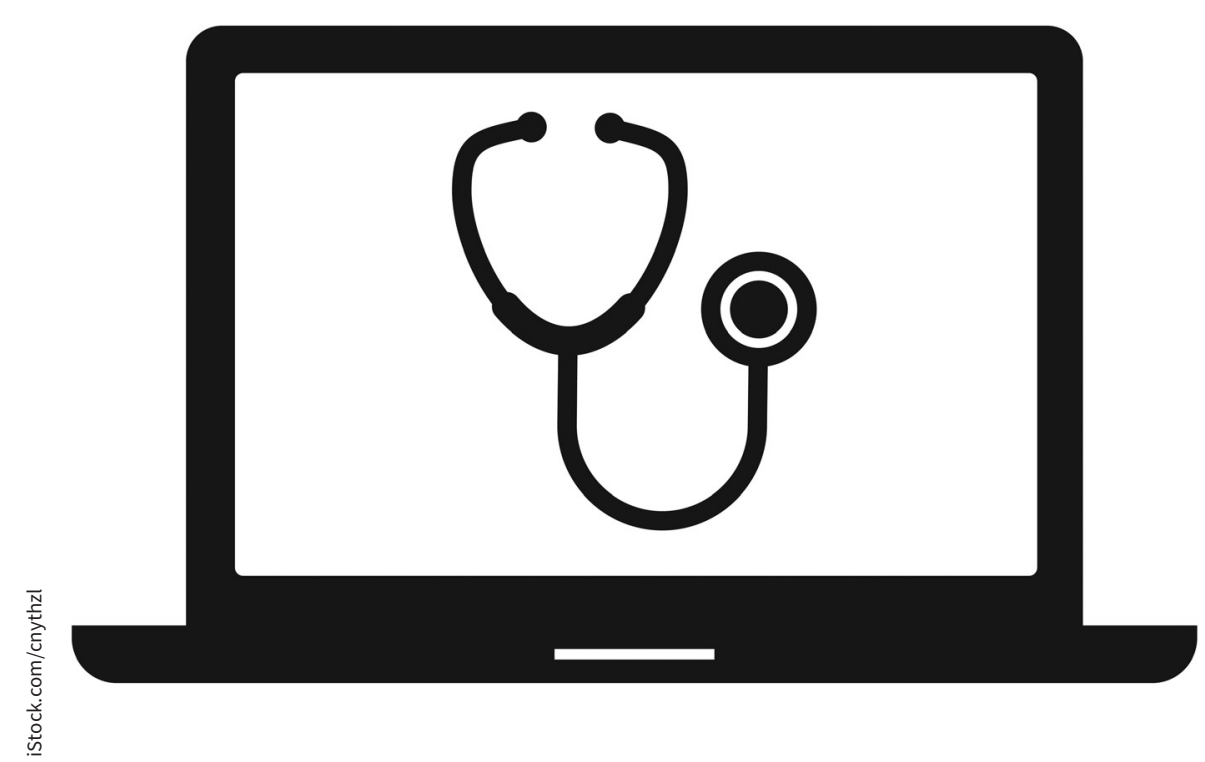

Many physicians think virtual care should be integrated into traditional primary care instead of operating outside the system.

small communities, testing is done at hospitals and ordering physicians need local hospital privileges. Virtual care physicians don't have those privileges, so patients are told to visit local doctors and request tests. "If the doctor in the community doesn't agree that the test should be done, it becomes a point of conflict between them and the patient," said Newbery.

The concerns are echoed by the Ontario Medical Association (OMA). "Virtual care offerings, including virtual walk-in clinics and apps, have the potential to fragment care and disturb integrated care," Dr. Sohail Gandhi, OMA president, said in a statement. He added that virtual care should be "provided as a supplement to - and not as a replacement of - traditional primary care."

According to Newbery, virtual walk-in clinics shouldn't be funded if communities have enough physicians to provide timely access to care for local patients. Instead, the government should incentivize family practices to offer virtual care in the medical home model by offering a system easy for patients to set up on their own devices.

Such a model has been piloted by the Ontario Telemedicine Network. Called Enhanced Access to Primary Care, it involves almost 200 doctors and more than 6,000 patients from five Local Health Integration Networks, according to Dr. Ed Brown, CEO of the network. Patients in the 
pilot are able to text doctors or talk by voice or video. Doctors are paid the same amount for e-consults as for in-person visits. Unlike with the walk-in model, patients communicate with their own family physicians, who can access their electronic medical records.

According to Brown, an evaluation completed by Women's College Hospital Institute for Health Systems Solutions and Virtual Care found that $98 \%$ of patients felt the visits were the same or better than inperson care. More than $90 \%$ of consults involved only text, and doctors frequently replied within a day or two. (The evaluation report has not yet been made public.)

Brown said the gold standard should be "primary care providers providing virtual care for their own patients," explaining that "continuity of care means better health outcomes and lower costs." The government's remuneration scheme could underlie the success of integration of virtual medicine into primary care. Brown said the independent evaluation recommended that the government continue the pilot study's practice of remunerating virtual consults the same as in-person visits.

According to David Jensen, media spokesperson for the Ontario Ministry of Health and Long-Term Care, the government is "currently reviewing the results of the Enhanced Access to Primary Care pilot, which will inform the provincial approach to modernizing virtual care in Ontario."

In the meantime, Liao said, Good Doctors is working to integrate care better and improve continuity, by connecting with family doctors to help them provide virtual care to patients who can't easily access their clinics, such as seniors in retirement homes. The model also aims to provide continuity by ensuring the same doctors are virtually available to patients on a set schedule each week, and that doctors follow up on tests and referrals, which may not occur in traditional walk-in clinics, said Liao.

Wendy Glauser, Toronto, Ont. 